\title{
Caso Radiológico Pediátrico
}

\author{
JORGE LLANOS C.* y CRISTIÁN GARCÍA B.****
}

\section{Historia Clínica}

Niño de 1 año 2 meses de edad, que estando en la cocina de su casa, es sorprendido por su madre en el momento en que su hermano mayor de 4 años, le daba a ingerir líquido de una botella. En ese momento, presenta episodio brusco de tos, que cede espontáneamente en menos de cinco minutos. Después de esto, la madre se percata que la botella contenía Kerosene (parafi- na), por lo que decide llevar al niño a un Servicio de Urgencia.

A su ingreso a Urgencia, el niño estaba en buenas condiciones generales, afebril, sin disnea ni polipnea. La faringe estaba congestiva y en el examen pulmonar, se auscultaban roncus y estertores basales bilaterales.

En ese momento, se solicitó una radiografía $(\mathrm{Rx})$ de tórax, en proyecciones anteroposterior y lateral (Figura 1).

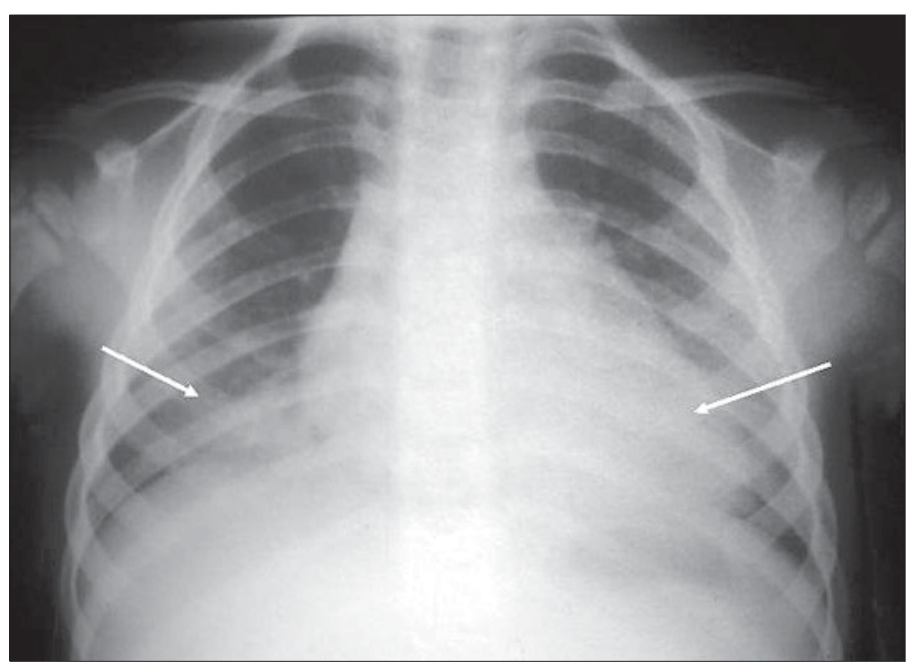

Figura 1a.

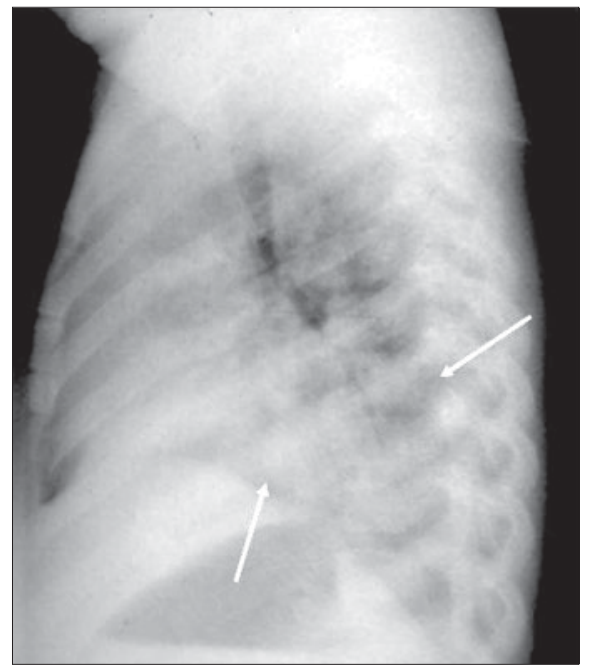

Figura $1 b$.

\section{¿Cuál es su diagnóstico?}

\section{¿Cuál sería la conducta terapéutica adecuada?}

\footnotetext{
* Departamento de Radiología, Facultad de Medicina, Pontificia Universidad Católica de Chile.

** Departamento de Pediatría, Facultad de Medicina, Pontificia Universidad Católica de Chile.
} 


\section{Hallazgos Radiológicos}

La Rx de tórax (Figura 1) muestra infiltrados confluentes en ambas bases pulmonares (flechas). No hay derrame pleural y el corazón no muestra alteraciones.

Considerando los antecedentes clínicos, estos hallazgos son compatibles con una neumopatía bilateral por aspiración de Kerosene.

El niño fue hospitalizado para observación y tratamiento antibiótico profiláctico. Evolucionó bien, sin complicaciones y fue dado de alta al tercer día, en buenas condiciones generales, asintomático.

\section{Diagnóstico}

Neumopatía bilateral por aspiración de Kerosene.

\section{Discusión}

Las intoxicaciones son una causa no infrecuente de morbimortalidad en la edad pediátrica y constituyen un importante problema de salud pública. Se ha informado que más de 1,5 millones de individuos menores de 19 años son afectados en EE.UU. anualmente, representando el $66 \%$ del total de intoxicaciones de ese país ${ }^{1}$. El mayor porcentaje de niños afectados es menor de 4 años y en más del $90 \%$ de los casos, es de causa accidental. De los menores de 6 años que sufren de intoxicaciones, 8,6 por 1.000 tendrán efectos severos o fatales ${ }^{1}$. Dentro de las sustancias con mayor morbimortalidad se encuentran drogas ilícitas, sedantes-hipnóticos, anticonvulsivantes, mordeduras, hidrocarburos, medicamentos cardiovasculares ${ }^{1}$. Según estadísticas nacionales manejadas por el Centro de Información Toxicológica de la Universidad Católica (CITUC), del total de llamadas recibidas por intoxicaciones, alrededor del 50\% corresponde a niños menores de 4 años, siendo la ingestión la vía de intoxicación más frecuente ${ }^{2}$. Más de la mitad son producidas por medicamentos, encontrando a los hidrocarburos como responsables en el $6 \%$ del total de intoxicaciones ${ }^{2}$.

La ingestión oral accidental de hidrocarburos tiene como consecuencia, principalmente una toxicidad pulmonar por aspiración, más que por absorción a nivel gastrointestinal. Aunque puede ocurrir compromiso del sistema nervioso central, renal, hepático o cardiovascular, la consecuencia más común y severa es la neumonitis química por aspiración ${ }^{3-5}$.

La extensión y severidad del compromiso pulmonar depende del tipo de hidrocarburo as- pirado y en especial, de su viscosidad. Al ingerir hidrocarburos, el riesgo de aspiración es inversamente proporcional a su viscosidad. Hidrocarburos de alta viscosidad y por ende de baja toxicidad son el asfalto, aceite mineral, lubricantes. Por otro lado, productos hidrocarbonados como gasolina o kerosene, poseen una baja viscosidad y difunden rápidamente y por lo tanto, tienen un alto riesgo de aspiración y de penetración profunda a los pulmones ${ }^{5,6}$.

El daño de la aspiración de hidrocarburos está dado por la neumonitis química y alteración del surfactante pulmonar. La manifestación clínica principal es una alteración en la relación ventilación-perfusión (V/Q), lo que se traduce en el desarrollo de hipoxemia y atelectasias. Otras potenciales consecuencias son la aparición de necrosis de áreas pulmonares y de derrame pleural, generalmente estériles. No es infrecuente que los pacientes que han aspirado estos productos desarrollen neumonías bacterianas por sobreinfección, por la disminución en la capacidad de clearance del pulmón ${ }^{3}$.

La histopatología de la aspiración de hidrocarburos se caracteriza por edema y acumulación de células inflamatorias con compromiso intraalveolar, intrabronquial, peribronquial e intersticial ${ }^{7,8}$.

El diagnóstico se sospecha principalmente basado en la clínica y los datos de las circunstancias en que ocurrió el evento. Se debe solicitar a la familia una muestra de la sustancia que se ingirió, como también el envase que lo contenía, para lograr un diagnóstico más preciso.

El examen físico inicial es muy importante. Se debe inspeccionar la mucosa oral ya que los efectos locales de la ingestión de hidrocarburos ocurren en forma inmediata. De esta manera, pueden observarse una importante irritación de la mucosa. La auscultación pulmonar inicial puede revelar solo disminución de ruidos pulmonares o roncus. Debido a que el compromiso pulmonar es progresivo, se debe monitorear en forma permanente la oximetría en busca de hipoxemia. De existir distrés respiratorio, éste se manifiesta en forma temprana con tos, disnea, quejidos. La aparición de fiebre no es infrecuente, y es secundaria a la respuesta inflamatoria producida. Síntomas neurológicos como temblor, confusión, convulsiones e incluso coma, se deberían a compromiso indirecto causado por hipoxemia más que a daño directo del sistema nervioso central $3,7,8$.

Dentro del estudio de laboratorio, la alteración V/Q se puede manifestar con hipoxemia, sin hipercarbia en los gases sanguíneos. Una 
hipoxia severa puede estimular la hiperventilación provocando una alcalosis respiratoria ${ }^{6}$.

El estudio inicial debe incluir una radiografía $(\mathrm{Rx})$ de tórax. Es frecuente que los hallazgos radiológicos no concuerden con la clínica, por mayor severidad de los cambios radiológicos, los que se hacen evidentes dentro de las primeras doce horas después de ocurrida la intoxicación. Inicialmente aparecen sombras confluentes de tipo alveolar en ambos pulmones, en especial en las bases, lo que puede asociarse a atelectasias y derrame pleural. El mayor compromiso pulmonar se evidencia dentro de las siguientes 72 horas al episodio y tiende a regresar dentro de los siguientes días a semanas ${ }^{7-9}$.

Dentro de las complicaciones más frecuentes, se incluye el desarrollo de neumatoceles, los cuales se producirían por la confluencia de áreas de necrosis bronquiolar, sumado al efecto de válvula que produce el engrosamiento de la pared bronquial. Estos aparecen típicamente después de que los síntomas clínicos ceden y pueden persistir por meses. Otra complicación frecuente es la aparición de derrame pleural de tipo inflamatorio. Complicaciones infrecuentes son abscesos pulmonares, formación de fístulas broncopleurales y neumotórax. En el caso de sospechar estas complicaciones el estudio radiológico simple se puede complementar con tomografía computarizada de tórax ${ }^{7-9}$.

El tratamiento de pacientes que han ingerido hidrocarburos dependerá de la severidad del daño pulmonar y/o sistémico ocasionado. La necesidad de hospitalización debe basarse en el tipo de hidrocarburo ingerido, alteraciones en el examen físico, oximetría de pulso y $\mathrm{Rx}$ de tórax. En el caso de niños que hayan ingerido productos de alta densidad y sin alteraciones físicas ni radiológicas, y en quienes no hay sospecha de negligencia de los padres, éstos pueden observarse por algunas horas y luego controlarse en forma ambulatoria. Los pacientes que hayan ingerido hidrocarburos de alto riesgo de aspiración, con alteraciones clínicas o radiológicas, deben ser hospitalizados para observación por al menos 12-72 h en centros que dispongan de cuidados intensivos pediátrico, debido al alto riesgo de progresión del daño pulmonar. En el caso de existir alteración severa de la función respiratoria, se debe hospitalizar al niño en una unidad de cuidados intensivos, ya que la inflamación, hipoxemia y falla pulmonar podría derivar en un síndrome de distrés respiratorio ${ }^{4-6}$.

El uso de eméticos o lavado gástrico generalmente está contraindicado, debido a que el principal mecanismo de intoxicación no es la absor- ción gastrointestinal, y al realizar estos procedimientos se podría favorecer el fenómeno aspirativo $^{10}$.

El uso de corticoides en la fase aguda, y antibióticos en forma profiláctica no ha demostrado mejorar el pronóstico de estos pacientes, como tampoco se ha visto beneficio terapéutico con el aporte de surfactante exógeno ${ }^{4,6}$.

Dentro de las complicaciones gastrointestinales de la ingestión de hidrocarburos, está la ulceración gástrica o esofágica, con eventual perforación ${ }^{6}$.

En los pacientes que sobreviven al daño pulmonar inicial, la disfunción pulmonar puede tomar años en recuperarse, lo cual debe controlarse con exámenes de función pulmonar.

Finalmente, no podemos olvidar la importancia de la educación para prevenir este tipo de accidentes, promoviendo el correcto uso y almacenaje de productos químicos y medicamentos en el hogar, en las escuelas, etc, en lugares seguros y fuera del alcance de los niños ${ }^{10}$.

\section{Bibliografía}

1.- LITOVITZ T, WHITE N, WATSON W. Epidemiology of Pediatric Poison Exposures: An Analysis of 2003 Poison Control Center Data. Clin Ped Emerg Med 2005; 6: 68-75.

2.- Centro de Información Toxicológica Universidad Católica de Chile (CITUC). Estadísticas. Memoria 2003.

3.- GENTINA T, TILLIE-LEBLOND I, BIROLLEAU S, SAIDI F, SAELENS T, BOUDOUX L, et al. Fire-eater's lung: seventeen cases and a review of the literature. Medicine (Baltimore) 2001; 80: 291-7.

4.- SEGEV D, SZOLD O, FIREMAN E, KLUGER Y, SORKINE P. Kerosene-induced severe acute respiratory failure in near drowning: reports on four cases and review of the literature. Crit Care Med 1999; 27: 143740.

5.- $\quad$ LING L J, CLARK R F, ERICKSON T B. Secretos de la toxicología. Mc Graw-Hill Editorial Interamericana 2002.

6.- Hospital infantil de México. Urgencias en pediatría. $5^{\mathrm{a}}$ ed. Mc Graw-Hill Editorial Interamericana, 2002.

7.- BRAY A, PIRRONI T, MARANO P. Pneumatoceles Following Hydrocarbon Aspiration. Eur Radiol 1998; 8: 262-3.

8.- FRANQUET T, GÓMEZ-SANTOS D, GIMÉNEZ A, TORRUBIA S, MONILL J M. Fire eater's pneumonia: radiographic and CT findings. J Comput Assist Tomogr 2000; 24: 448-50.

9.- KIM K I, KIM C W, LEE M K, LEE K S, PARK C K, CHOI S J, et al. Imaging of Occupational Lung Disease. Radiographics 2001; 21: 1371-91.

10.- SHANNON M. Ingestion of Toxic Substances by Children. N Engl J Med 2000; 342: 186-91.

Correspondencia a:

Jorge Llanos C.

E-mail: jgllano@puc.cl 